\section{Theoretical Calculations on Solid Argon}

IT is well known that a knowledge of the second virial coefficient of a gas does not suffice to specify the intermolecular potential of the constituent molecules. In fact, even if a simple formula of MieLennard-Jones type is used as an approximation to the potential, the parameters are not uniquely determined by the second virial coefficient. On the other hand (as mentioned in the preceding communication) certain elementary properties of the solid state are sufficient to determine these parameters uniquely, and further observations on the solid and gaseous states can then be used as a test of the region of validity of this type of approximation to the potential. The recent development of experimental technique enabling data on the solid state to be determined with accuracy over a wide range of pressures and tem. peratures has provided valuable material for theoret. ical analysis.

When the isotherms for solid argon at high pressures determined by Stewart ${ }^{1}$ at the Massachusetis In stitute of Technology became available, one of us (I. J. Z. ${ }^{2}$ ) undertook detailed calculations to determine how the results compared with the theoretical predictions, and, if necessary, to modify the estimates of intermolecular potentials for the inert gases concerned. Although quite good agreement was obtained, the form of potential used was not completely satisfactory because of the uncertainty in the value of the density at absolute zero. The present accurate experimental results obtained at Queen Mary College for argon enable this uncertainty to be removed, and further detailed comparisons to be made between theory and experiment for this substance.

We have used the following approximation to the intermolecular potential energy :

$$
\begin{aligned}
\varepsilon(r) & =\lambda / r^{12}-\mu / r^{8} \\
\lambda & =1.63 \times 10^{-7} \mathrm{A.} .^{12} \mathrm{erg} . \\
\mu & =1.05 \times 10^{-10} \mathrm{A.} .^{6} \mathrm{erg} .
\end{aligned}
$$

To calculate the thermal expansion and Gruneisen's $\gamma\left(=\alpha V / x_{T} C_{v}\right.$ or $\left.\alpha V / x_{8} C_{p}\right)$, we have used the approximation of Henkel ${ }^{3}$. This approximation is of Einstein type but takes account also of anharmonic effects ; it should be reliable at temperatures not too low compared with the Debye $\theta$ value. The results are as follows :

$$
\begin{aligned}
& T\left({ }^{\circ} \mathrm{K} .\right) \\
& \rho\left(\mathrm{gm} . \mathrm{cm}^{-3}\right) \\
& a \times 10^{4}\left(\mathrm{deg}^{-1} \mathrm{C} .\right) \\
& \gamma
\end{aligned}
$$

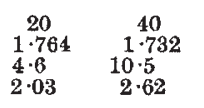

$\begin{array}{cc}60 & 80 \\ 1 \cdot 690 & 1 \cdot 634 \\ 14 \cdot 6 & 20 \cdot 0 \\ 2 \cdot 86 & 2 \cdot 50\end{array}$

These are to be compared with the experimental values of the preceding communication.

It will be seen that the theoretical calculations are in good agreement with the experimental results. Calculations at higher pressures based on the above estimate of the intermolecular potential are also in good agreement with experimental isotherms given by Stewart ${ }^{1}$. A further check on the estimate of the potential is provided by the Debye $\theta$ value for solid argon at low temperatures. This can be calculated approximately and simply for a potential of the form (1) and yields the value of $80^{\circ} \mathrm{K}$., in good agreement with the experimental value derived by R. W. Hill ${ }^{5}$ from measurements of the specific heat of solid argon at low temperatures. The values of $\lambda$ and $\mu$ given in (1) are very close to those determined recently by Whalley and Schneider ${ }^{6}$ from data for gaseous argon.

We may thus conclude that theoretical calculations of solid-state data based on the intermolecular petential (1) give results in reasonable agreement with experiment. Further experimental data, particularly at high pressures, would be desirable to provide a more searching test of the theory.

\section{Dомв}

\section{J. ZuCKeR}

Wheatstone Laboratory, King's College,

London, W.C.2. July 30.

'Stewart, J. W., Phys. Rev., 97, 578 (1955).

2 Zucker, I. J., J. Chem. Phys. (in the press).

${ }^{3}$ Henkel, J. H., J. Chem. Phys., 23, 681 (1955).

4 Domb, C., and Salter, L. S., Phil. Mag., 43, 1083 (1952).

- Hill, R. W., thesis, Oxford (1952).

${ }^{6}$ Warlley, E., and Schnelder, W. G., J. Chem. Phys., 23, 1644 (1955).

\section{Nuclear Spin of Americium-24I}

DURING the recording of the spark spectrum of americium ${ }^{1}$ in the third order of the Baird three-metre grating spectrograph, a number of spectral lines appeared as four resolved components of a probable six-member flag pattern. While describing the performance of a $30-\mathrm{ft}$. Paschen-Runge spectrograph, Tomkins and Tomkins ${ }^{2}$ have published a photograph of the Am I line at $6405 \mathrm{~A}$. showing it resolved into six components. The six component flag patterns, which lead to a nuclear spin of $5 / 2$ for ${ }_{95} \mathrm{Am}^{241}$, have been confirmed by interferometric studies.

In the present investigation americium was excited in a hollow cathode cooled with liquid nitrogen. High dispersion was achieved by crossing a Hilger Universal Prism spectrograph with Fabry-Perot étalon plates coated with $\lambda / 4$ layers of cryolite and zinc sulphide. A series of different spacers were used for eliminating erior's due to overlapping orders. Fig. 1 shows the six components of the Am I lines at $4662 \cdot 80$ and $4681 \cdot 67$, the separations between the components being $0.230 \pm 0.0015 \mathrm{~cm}^{-1}, 0.212 \pm 0.001 \mathrm{~cm}^{-1}$, $0 \cdot 184 \pm 0.0015 \mathrm{~cm}^{-1}, 0 \cdot 147 \pm 0.002 \mathrm{~cm}^{-1}, 0.104 \pm$ $0.003 \mathrm{~cm} .^{-1}$, and $0.274 \pm 0.001 \mathrm{~cm}^{-1}, 0.226 \pm$ $0.002 \mathrm{~cm}^{-1}, \quad 0.180 \pm 0.001 \mathrm{~cm}^{-1}, \quad 0.138 \pm 0.002$ $\mathrm{cm}^{-1}, 0.101 \pm 0 \cdot 002 \mathrm{~cm} .^{-1}$, respectively.

Since these and other hyperfine separations show a marked divergence from the interval rule, a relatively large quadrupole-moment is expected for americium.

On the assumption that the $5 f_{5 / 2}$ shell is filled preferentially to the $5 f_{\eta t 2}$, the value of a nuclear spin of $5 / 2$ for ${ }_{95} \mathrm{Am}^{241}$ is in accordance with Klinkenberg's ${ }^{3}$ nuclear shell structure tables which are based on

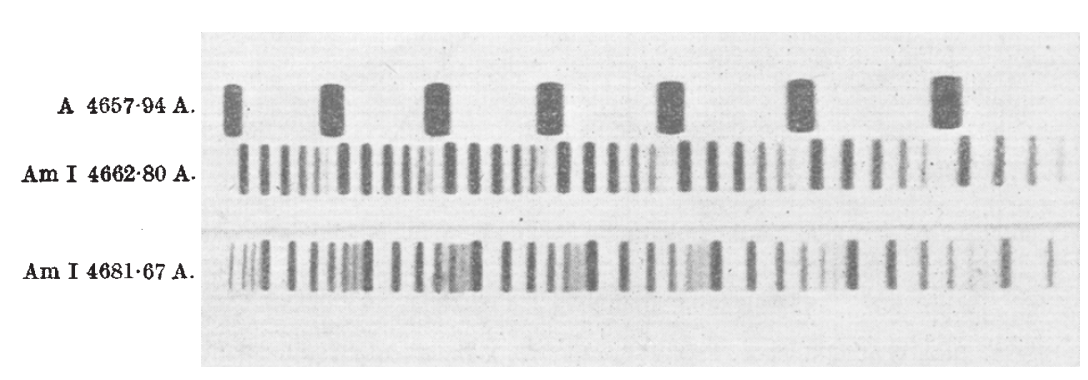

Fig. 1. Hyperflne structure of Am I lines at $4662 \cdot 80$ and $4681 \cdot 67 \mathrm{~A}$. 